Revista Brasileira de Agricultura Irrigada v.10, no .3, p. 631 - 639, 2016

ISSN 1982-7679 (On-line)

Fortaleza, CE, INOVAGRI - http://www.inovagri.org.br

DOI: $10.7127 /$ rbai.v10n300385

Protocolo 385.16 - 03/12/2015 Aprovado em 13/06/2016

\title{
PARAMETRIZAÇÃO DO MODELO DSSAT/CANEGRO PARA VARIEDADES DE CANA-DE-AÇÚCAR EM ALAGOAS
}

\author{
Allan Cunha Barros ${ }^{1}$, José Leonaldo Souza ${ }^{2}$, Rubens Duarte Coelho ${ }^{3}$, Alexandre Hugo César \\ Barros $^{4}$, Alexsandro Cláudio dos Santos Almeida ${ }^{5}$, Jessé Marques da Silva Junior ${ }^{6}$
}

\section{RESUMO}

O modelo DSSAT/CANEGRO não disponibiliza em seu banco de dados parametrização para variedades brasileiras, limitando seu uso para no Brasil. Assim, o objetivo desse trabalho foi parametrizar quatro variedades de cana-de-açúcar plantadas no Estado de Alagoas utilizando o modelo DSSAT/CANEGRO: RB92579, RB93509, SP79-1011 e RB931530. As simulações foram realizadas na Universidade Federal de Alagoas (UFAL), Campus Arapiraca. Os dados para as simulações foram provenientes de um experimento de cana-planta conduzido por Almeida et al. (2008) no Centro de Ciências Agrárias da UFAL, onde foram obtidos os dados biométricos das variedades (produtividade de massa seca e fresca, índice de área foliar (IAF), número de perfilhos e altura de planta), para as variedades RB92579, RB93509, SP79-1011 e RB931530 . O desempenho do modelo foi avaliado por meio dos índices estatísticos: índice de concordância (d), raiz quadrada do erro médio (RMSE), índice de confiabilidade (IC), erro absoluto médio (MAE) e o coeficiente de determinação $\left(\mathrm{R}^{2}\right)$. Os resultados mostram que após as calibrações das variedades, os índices estatísticos obtiveram, em média, valores ótimos (d $>0,85)$ para o IAF e número de perfilhos, e muito bom $(0,76<\mathrm{d}<0,85)$ para a produtividades de massa fresca e seca, indicando que os valores observados e estimados foram próximos, com

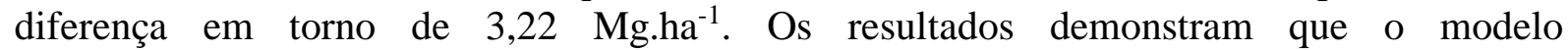
DSSAT/CANEGRO foi capaz de descrever adequadamente a produtividade da cana-de-açúcar para as 4 variedades estudadas.

Palavras-chave: Saccharum officinarum, modelo de simulação, setor sucroenergético

\footnotetext{
${ }^{1}$ Eng. Agrônomo. Professor do Curso de Agronomia da UFAL, Campus Arapiraca. Av. Manoel Severino Barbosa, Bom Sucesso, Arapiraca, AL, Brasil. email: allan.cunha.barros@gmail.com

${ }^{2}$ Agrometeorologista. Professor do curso de Agrometeorologia da UFAL. Av. Lourival Melo Mota, s/n

Tabuleiro dos Martins Maceió, AL, Brasil. email: jls@ccen.ufal.br

${ }^{3}$ Eng. Agrônomo. Professor do Departamento de Engenharia de Biossistemas, ESALQ/USP. Av. Pádua Dias, 11, Piracicaba, SP, Brasil. email: rdcoelho@usp.com

${ }^{4}$ Eng. Agrônomo. Pesquisador da Embrapa Solos. R. Antônio Falcão, 402 - Boa Viagem, Recife, PE, Brasil. email: alex.hugo.2006@gmail.com

${ }^{5}$ Eng. Agrônomo. Professor do Curso de Agronomia da Faculdade de Ciências Agrárias, UFGD. Rodovia Dourados - Itahum, Km 12 - Cidade Universitária, Dourados - MS,Brasil. email: alexsandroalmeid@ufgd.edu.br

${ }^{6}$ Eng. Agrônomo. Bolsista Pós-doutorado da UFAL, Campus Arapiraca. Av. Manoel Severino Barbosa, Bom Sucesso, Arapiraca, AL, Brasil. email: jesseagronomo@yahoo.com.br
} 


\title{
PARAMETIZATION OF DSSAT/CANEGRO VARIETY OF SUGARCANE IN ALAGOAS
}

\begin{abstract}
The DSSAT/CANEGRO's database there aren't available parameterization with Brazilian varieties, limiting its use in Brazil. Then, the aim of this study was to parameterize four varieties of sugarcane planted in the State of Alagoas using the model DSSAT/CANEGRO. The simulations were performed at the Federal University of Alagoas (UFAL), Campus Arapiraca. The experimental data for the simulations were from the experiment conducted by Almeida et al. (2008), in Agricultural Sciences Center at UFAL, which were obtained biometric data of varieties (fresh and dry matter productivity, leaf area index (LAI), number and plant height) for the varieteis RB92579, RB93509, SP791011 e RB931530. The model performance was evaluated by means of statistical indices: index of agreement (d), square root of the mean error (RMSE), reliability index (CI), mean absolute error (MAE) and the coefficient of determination $\left(\mathrm{R}^{2}\right)$. The results show that after the calibration of varieties, the statistical indices obtained for the IAF and numbers of tillers were "optimum values" ( $d>0.85$ ); for the yield of fresh and dry weight obtained were "very good" $(0,76<\mathrm{d}<0,85)$. The yield observed was close to the estimated values of yield, the difference was almost 3.22 Mg.ha' ${ }^{-1}$. This results showed that model DSSAT/CANEGRO was able to adequately describe the sugarcane yield for 4 studied varieties.
\end{abstract}

Keyword: Saccharum officinarum, model simullation, sugarcane industry

\section{INTRODUÇÃO}

O Brasil é um dos principais celeiros agrícolas do mundo e considerado o principal exportador de açúcar derivado da cana-deaçúcar. A cadeia sucroenergética dessa cultura representa um dos principais setores econômicos no Estado de Alagoas, com grande geração de empregos e renda. Representa a principal atividade econômica nas regiões dos Tabuleiros Costeiros e Zona da Mata do Estado, regiões tradicionalmente canavieiras, que não dispõem mais de área para sua expansão (ALMEIDA et al. 2008). Dessa forma, o aumento de produção dependerá exclusivamente das adoções de tecnologias de cultivo e gestão que possam incrementar a produtividade agrícola.

Nesse sentido, a modelagem é uma importante ferramenta no planejamento e tomadas de decisão das atividades agrícolas, sendo assim existem dois tipos de esferas interessadas nestas informações estratégicas: a) a empresa sucroenergética para aperfeiçoar suas programações operacionais da época ideal de corte e planejamento agrícola da lavoura canavieira - o que é fundamental na otimização da rentabilidade do empreendimento (SCARPARI; BEAUCLAIR, 2004, 2009); b) o governo que se utiliza do zoneamento edafoclimático da cultura para minimizar o risco de sinistro no sistema de financiamento bancário (ROSSETTI, 2001).

Na cultura da cana-de-açúcar, exemplos da utilização de modelos incluem zona agroecológica com sucesso para previsão da safra da cana-de-açúcar em regiões do estado de Minas Gerais (OLIVEIRA et al., 2012; OLIVEIRA et al., 2013).

O modelo APSIM, desenvolvido pela CSIRO (Commonwealth Scientific and Industrial Research Organisation) (O'LEARY et al., 1999) foi calibrado para avaliar o efeito da palhada na produtividade (COSTA, 2012) e do nitrogênio na produtividade da cana-deaçúcar em Jaú-SP (URIBE, 2009).

Outra plataforma que vem sendo adotada internacionalmente é o DSSAT (Decision Support System for Agrotechnology Transfer), no entanto as variedades de cana-de-açúcar cadastradas no banco de dados do DSSAT/CANEGRO são originarias da 
Austrália e África do Sul, portanto, são diferentes das cultivadas no Brasil. Marin et al. (2011) foram um dos primeiros a calibrar variedades de cana-de-açúcar brasileiras no modelo DSSAT/CANEGRO, utilizado as variedades SP 83-2847 e RB 72-454, cultivadas nas condições da região sudeste do país.

Nassif et al. (2012), buscando aumentar o número de calibrações para o modelo DSSAT/CANEGRO, calibraram as variedades: CTC 4, CTC 7 e CTC 10, além das RB 86-7515 e RB 83-5486, para a região de São Paulo. Silva (2012) calibrou a cultivar RB 92-579, para as condições de clima e solo de Petrolina-PE. Apesar disso, há uma grande gama de variedades de cana-de-açúcar sem calibração para o modelo DSSAT/CANEGRO, e as já calibradas, foram, em sua maioria, realizadas para a região sudeste do Brasil.

Diante do exposto, faz-se necessário a calibração de novas variedades potenciais e já cultivadas na região. Assim, o objetivo desse trabalho foi parametrizar quatro variedades de cana-de-açúcar, utilizando o modelo DSSAT/CANEGRO, para as condições do Estado de Alagoas.

\section{MATERIAL E MÉTODOS}

O estudo foi desenvolvido na Universidade Federal de Alagoas, Campus Arapiraca, em parceria com a EMBRAPA Solos e ESALQ/USP. As parametrizações foram realizadas utilizando-se o modelo de simulação CANEGRO, que faz parte do pacote de modelos no sistema computacional DSSAT (Decision Support System for Agrotechnology Transfer).

As variedades parametrizadas foram: RB92-579, RB93-509, SP79-1011 e RB931530, cujos dados experimentais foram coletados de Almeida, et al. (2008), em trabalho desenvolvido na área experimental de Agrometeorologia do Centro de Ciências Agrárias da Universidade Federal de Alagoas, Campus Delza Gitaí, Rio Largo, (09²802"S; 354943"W; $127 \mathrm{~m}$ ), região dos Tabuleiros Costeiros de Alagoas. O solo do experimento foi um Latossolo Amarelo coeso argissólico de textura média/argilosa.

Para a parametrização do modelo são necessários dados de clima, solo e variedade. Assim, o conjunto das variáveis meteorológicas diárias de entrada utilizadas correspondeu aos valores diários de temperatura máxima e mínima do ar $\left({ }^{\circ} \mathrm{C}\right)$, precipitação pluviométrica $(\mathrm{mm})$ e radiação solar (MJ m${ }^{-2} \operatorname{dia}^{-1}$ ), que foram coletadas na estação meteorológica automática localizada na mesma área do experimento.

As variáveis biométricas das plantas, coletadas por Almeida, et al. (2008), foram: número de perfilhos (número de colmos. $\mathrm{m}^{-2}$ ), altura do colmo (m), índice de área foliar (IAF), além da produtividade de colmos, massa fresca e seca (Mg.ha-1), e açúcar (Mg.ha ${ }^{-}$ 1). As avaliações do perfilhamento e altura dos colmos foram realizadas a cada quinze dias e o IAF foi medido mensalmente. O delineamento experimental utilizado foi 0 de blocos casualizados, com cinco repetições e 4 tratamentos. O experimento foi conduzido numa área de $500 \mathrm{~m}^{2}$, subdividida em parcelas de cinco linhas de cultivo com 4 metros lineares (ALMEIDA, et al. 2008). O ciclo da cultura foi de outubro de 2003 a novembro de 2004.

Nassif et al. (2012) avaliaram a sensibilidade do modelo DSSAT e determinaram quais os parâmetros que mais influenciam na calibração da cana-de-açúcar, assim determinou-se os parâmetros que seriam otimizados no momento da parametrização (Tabela 1). As variáveis de saída utilizadas foram: IAF, número de perfilhos, a altura de planta, produtividade de massa fresca e seca, e produtividade de açúcar.

Tabela 1. Parâmetros de variedades utilizados na calibração do modelo DSSAT/CANEGRO, segundo Nassif et al. (2012).

Parâmetro Unidade Descrição




\begin{tabular}{|c|c|c|}
\hline Parcemáx & g.MJ $\mathrm{MJ}^{-1}$ & $\begin{array}{l}\text { Máxima eficiência na conversão de radiação expressa em } \\
\text { assimilados produzidos antes da respiração, por unidade de radiação } \\
\text { fotossinteticamente ativa (PAR) }\end{array}$ \\
\hline APFMX & Mg. $\mathrm{Mg}^{-1}$ & $\begin{array}{c}\text { Fração máxima de incremento de massa de matéria seca que pode } \\
\text { ser alocado para massa de matéria seca da parte aérea }\end{array}$ \\
\hline STKPFmáx & Mg.Mg-1 & $\begin{array}{l}\text { Fração do incremento diário de massa de matéria seca aérea } \\
\text { particionada para o colmo em altas temperaturasem cultura madura }\end{array}$ \\
\hline Suca & Mg. $\mathrm{Mg}^{-1}$ & Máxima sacarose contida na base do colmo \\
\hline TBFT & ${ }^{\circ} \mathrm{C}$ & $\begin{array}{l}\text { Temperatura em que o particionamento para sacarose é } 50 \% \text { do } \\
\text { máximo incremento de massa de colmo sem estresse }\end{array}$ \\
\hline Tthalfo & GD & $\begin{array}{l}\text { Graus-dia para que o desenvolvimento do dossel atinja metade do } \\
\text { espaçamento }\end{array}$ \\
\hline Tbase & ${ }^{\circ} \mathrm{C}$ & Temperatura base para o desenvolvimento do dossel \\
\hline LFmáx & Folhas & Número máximo de folhas verdes saudáveis \\
\hline MXLFArea & $\mathrm{cm}^{2}$ & Área foliar máxima atribuída às folhas sobre o número de folhas \\
\hline MXLFArno & Folha & $\begin{array}{l}\text { Número da folha acima do qual a área foliar é limitada pelo } \\
\text { MXLFArea }\end{array}$ \\
\hline Pl1 & GD & Intervalo de filocrono 1 para as folhas abaixo do Pswitch \\
\hline $\mathrm{Pl} 2$ & GD & Intervalo de filocrono 2 para as folhas acima do Pswitch \\
\hline Pswitch & Folha & Número da folha em que há mudança no filocrono \\
\hline TTPLNTEM & GD & Graus-dia para emergência da cana-planta \\
\hline TTRATNEM & GD & Graus-dia para emergência da soqueira \\
\hline ChupiBase & GD & Graus-dia para início do crescimento do colmo \\
\hline TT_PopGrowth & GD & Graus-dia para ocorrer o pico do perfilhamento \\
\hline Max_Pop & Colmos.m $\mathrm{m}^{-2}$ & População de perfilhos máxima \\
\hline PopTT16 & Colmos.m $\mathrm{m}^{-2}$ & População de colmos após 1.600 graus-dia \\
\hline LG_AMBase & Mg.ha-1 & Massa de matéria fresca da parte aérea onde ocorre \\
\hline
\end{tabular}

Utilizou-se o procedimento de tentativa e erro por análise visual, mesmo procedimento utilizado por (COSTA, 2013), para o modelo APSIM, os dados gerados pelo programa foram trabalhados em planilha eletrônica e confeccionados os gráficos. Não se utilizou a ferramenta GLUE ("generalized likelihood uncertainty estimation”) (MERTENS et al., 2004), utilizada por Nassif et al. (2012), já que, segundo os autores, o método utiliza para o ajuste das variáveis apenas dados finais do ciclo da cultura e desprezava os dados coletados durante o seu desenvolvimento.

A qualidade da calibração foi avaliada através dos índices estatísticos: índice de concordância de Wilmot (d), raiz quadrada do erro médio (RMSE), índice de confiabilidade (IC) e o coeficiente de correlação de Pearson (r).
Utilizou a calibração da RB-92579 desenvolvida por Silva (2012), como ponto de partida para as calibrações realizadas para Alagoas, o critério para a adoção dessa calibração foi a proximidade da cidade de Petrolina - PE, local onde Silva desenvolveu sua calibração, e latitude próxima à região de estudo.

\section{RESULTADOS E DISCUSSÃO}

\section{Parametrização}

Na Tabela 2 é observada a qualidade da calibração dos parâmetros número de perfilhos, índice de área foliar, altura de planta.

Para o número de perfilhos, os índices demonstraram que a calibração variou de muito boa $(0,75$ a 0,85$)$ a ótima $(>0,85)$, pelo índice “d”, e de alta $(0,5$ a 0,7$)$ a muito alta $(0,7$ 
a 0,9) pelo índice " $r$ ". Os melhores resultados foram obtidos para a RB 92-579, e os que não se ajustaram muito bem, para RB 93-509. A variedade RB 93-509 possui como característica de crescimento baixo número de perfilhos, com diâmetro e massa de colmos maior, por isso os resultados apresentaram baixos índices. O IC variou de 0,56 a 0,8 .

O erro médio absoluto foi inferior a 2,26 perfilhos. $\mathrm{m}^{-2}$, valores semelhantes aos encontrados por Silva (2012), que obteve RMSE de 2,66 perfilhos.m ${ }^{-2}$, e inferiores aos encontrados por Nassif et al. (2012). Esses resultados, mais baixos, podem ter ocorrido pela utilização da calibração de Silva (2012) como base, do trabalho atual, já que a mesma possuía valor baixo e foi melhorado, ou pelo fato das calibrações desenvolvidas com menor valor de RMSE terem sido desenvolvidas pela metodologia da análise por tentativas.

O número de perfilhos tem relação com a produtividade e a capacidade do modelo prever tal índice e seus os efeitos ambientais, como a falta de água, são importantes para a previsão da safra. Por isso a qualidade deste parâmetro tem relação importante nas simulações, principalmente para plantios em sequeiro. Barbosa et al. (2014) avaliando a redução de perfilhos em relação ao déficit hídrico observaram que as variedades a RB867515 e SP81-3250 não diferiram estatisticamente.

Tabela 2. Qualidade da calibração dos parâmetros número de perfilhos, índice de área foliar, altura de planta.

\begin{tabular}{ccccc}
\hline Parâmetro & \multicolumn{5}{c}{ Perfilhos } \\
\hline & RB & SP & RB & RB \\
d & $92-579$ & $79-1011$ & $93-1530$ & $93-509$ \\
\cline { 2 - 5 } IC & 0,91 & 0,86 & 0,83 & 0,77 \\
r & 0,80 & 0,62 & 0,56 & 0,57 \\
RMSE $\left(n^{\circ} \cdot m^{-2}\right)$ & 0,88 & 0,72 & 0,68 & 0,74 \\
\cline { 2 - 5 } d & 0,82 & 1,42 & 1,65 & 2,26 \\
\cline { 2 - 5 } IC & 0,93 & 0,97 & 0,89 & 0,85 \\
r & 0,95 & 0,93 & 0,81 & 0,73 \\
RMSE (m $\left.{ }^{2} \cdot \mathrm{m}^{-2}\right)$ & 0,20 & 0,16 & 0,91 & 0,14 \\
\cline { 2 - 5 } d & 0,89 & 0,95 & 0,96 & 0,28 \\
IC & 0,21 & 0,12 & 0,11 & 0,14 \\
r & 0,75 & 0,88 & 0,89 & 0,88 \\
RMSE (m) & 0,84 & 0,93 & 0,93 & 0,94 \\
\hline
\end{tabular}

No modelo DSSAT/CANEGRO a fração de luz interceptada pela cultura é determinada pelo índice de área foliar, que é calculada pelo produto da área média de folhas verdes por perfilho e a população de perfilhos (Singels \& van der Berg, 2008). Barros (2011), utilizando a calibração desenvolvida por Marin et al. (2009) para a cultivar RB83-2847 (índice d de 0,79), observou que o modelo tem bastante sensibilidade para o parâmetro IAF, em que o mesmo atingiu o valor $0 \%$ quando induzido um estresse hídrico.

O índice de área foliar (IAF) foi a variável que apresentou os melhores resultados em relação às outras variáveis. O IC foi acima de 0,7, para a RB 93-509, e acima de 0,81 para as outras variedades de cana-de-açúcar. Pelo índice “d”, a calibração obteve como critério “ótima”, e pelo "r" ficou entre ótima e quase perfeita. Esses resultados também foram obtidos par as 
variedades CTC 4, CTC 7 e CTC 10 (NASSIF et al. 2012) e para a RB 92-579, cana soca (SILVA, 2012). O RMSE foi de no máximo $0,28 \mathrm{~m}^{2} \cdot \mathrm{m}^{-2}$ e a cultivar com melhor ajuste foi a SP 79-1011.

Apesar do IC baixo, valores menores que 0,21, a altura de colmo apresentou calibração ótima, segundo o índice “d”, e de muito alta a quase perfeita, pelo índice " $r$ ", com RMSE próximo de 0,9 m. Essa variação pode ter ocorrido devido a um estresse hídrico sofrido pela cultura como relatam Almeida et al. (2008), entre os decêndios 14 e 18, fazendo com que houvesse uma variação do IC da cultura, assim, o modelo não assumiu tal comportamento no momento da simulação.

Devido à limitação de dados disponíveis, não foi possível realizar a mesma análise estatística dos parâmetros acima, e sim, somente uma comparação de médias, o mesmo ocorreu no trabalho de Nassif et al. (2012) para quatro das cinco variedades calibradas. Apesar disso, pode ser visto na Tabela 3 as produtividades de massa fresca e açúcar das variedades RB.

Tabela 3. Tabela de produtividade da massa fresca observada e estimada pelo modelo DSSAT/CANEGRO, para a região de Alagoas.

\begin{tabular}{|c|c|c|}
\hline \multirow[t]{2}{*}{ Variedade } & \multicolumn{2}{|c|}{ Produtividade (Mg.ha-1) } \\
\hline & Observada * & Estimada \\
\hline RB 92-579 & 167,89 & 165,43 \\
\hline RB 93-509 & 110,30 & 115,54 \\
\hline SP79-1011 & 100,57 & 102,40 \\
\hline RB 93-1530 & 83,92 & 87,28 \\
\hline
\end{tabular}

Nota-se que os valores de produtividade observadas de massa fresca são muito próximos dos valores estimados pelo modelo DSSAT/CANEGRO, com variação média de

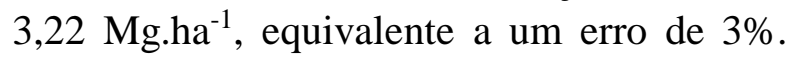
Oliveira et al. (2013), utilizando o modelo de Stewart para a estimativa da produtividade de cana-de-açúcar em Jaíba-MG, obteve diferenças entre as produtividades, observadas e estimadas, que variaram de 16,7 a $0,16 \%$, na safra 2008/2009.
Silva (2008) obteve alto coeficiente "d" $(0,98)$ para a estimativa do parâmetro de biomassa seca dos colmos, para a RB 92-579, cana soca, durante todo o ciclo da cultura, demonstrando o grande potencial que o modelo possui.

Na Tabela 4 são observados parâmetros das calibrações para cana-planta, na região de Alagoas, para as variedades: RB92-579, RB93-509, SP79-1011 e RB93-1530, comparada a variedade padrão e a calibrada por Silva (2012), a RB 92-579.

Tabela 4. Parâmetros calibrados para as variedades de cana-de-açúcar RB92-579, RB93-509, SP791011 e RB93-1530, após a calibração.

\begin{tabular}{lcccccc}
\hline & NCo & RB & RB & SP & RB & RB \\
& 376 & $92-579^{*}$ & $92-579$ & $79-1011$ & 1530 & $93-509$ \\
\hline Parcemáx & 9,90 & 10,8 & 13,5 & 7,7 & 6,5 & 9,86 \\
APFMX & 0,88 & 0,92 & 0,9 & 0,88 & 0,9 & 0,8 \\
STKPFmáx & 0,650 & 0,88 & 0,88 & 0,55 & 0,55 & 0,69 \\
Suca & 0,580 & 0,57 & 0,57 & 0,58 & 0,58 & 0,68 \\
TBFT & 25 & 25 & 25 & 25 & 25 & 25 \\
Tthalfo & 250 & 286 & 230 & 250 & 250 & 250,8 \\
Tbase & 16 & 14 & 14 & 15 & 14 & 15,71 \\
LFmáx & 12 & 8 & 8 & 12 & 12 & 9
\end{tabular}


ALAGOAS

\begin{tabular}{lcccccc} 
MXLFArea & 360 & 792 & 680 & 380 & 680 & 435 \\
MXLFArno & 15 & 22 & 14 & 14 & 14 & 14 \\
Pl1 & 69 & 109 & 65 & 90 & 90 & 110 \\
Pl2 & 169 & 117 & 179 & 179 & 179 & 200 \\
Pswitch & 18 & 22 & 18 & 18 & 18 & 14 \\
TTPLNTEM & 428 & 428 & 615 & 628 & 628 & 628 \\
TTRATNEM & 203 & 620 & 203 & 203 & 203 & 290 \\
ChupiBase & 1050 & 1050 & 533 & 1050 & 1050 & 855 \\
TT_PopGrowth & 600 & 628 & 789 & 700 & 700 & 800 \\
Max_Pop & 30 & 28 & 28 & 15 & 16 & 19,7 \\
PopTT16 & 13,3 & 12 & 11 & 9,2 & 7,8 & 8,3 \\
LG_AMBase & 220 & 220 & 220 & 200 & 200 & 220 \\
\hline
\end{tabular}

*Variedade padrão utilizada para efeito de comparação.

A maioria dos parâmetros obtidos para as variedades calibradas foram diferentes da NCo 376, o mesmo ocorreu em Marin et al. (2011) e Nassif et al. (2012). A calibração realizada por Silva (2012) diferiu da realizada nesse estudo. O principal motivo foi que a calibração realizada por Silva (2012), na cidade de Petrolina-PE, foram utilizados dados de cana soca (segundo corte da cana-de-açúcar), e nesse trabalho foram utilizados dado de cana planta.

Dentre as 20 variáveis utilizadas na parametrização, somente TTPLNTEM e TTRATNEM são específicas para cana-planta e cana-soca, além disso, no momento da criação dos cenários no programa DSSAT/CANEGRO apenas uma variável diferencia a cana planta da cana soca, que é o tipo de material que de onde a simulação deve iniciar, se por cana-planta ou cana-soca, no entanto, a cana planta e cana soca possuem diferentes comportamentos de crescimento. Corroborando esse argumento, Almeida et al. (2008) observaram diferenças no IAF, produção de colmos, perfilhamento e altura de colmo para as variedades calibradas nesse trabalho. Dessa forma, acredita-se que seja necessário realizar calibração diferenciada para a cana planta e soca. A parametrização foi realizada para cana planta, havendo necessidade de realizar a calibração para cana soca.
.Foi possível parametrizar as variedades de cana: RB92579, RB93509, SP79-1011 e RB931530, com calibrações avaliadas em muito boa a ótima, pelo índice “d”, e alta a muito alta pelo índice " $r$ ".

\section{AGRADECIMENTOS}

À Fundação de Amparo à Pesquisa do Estado de Alagoas pelo apoio financeiro.

\section{REFERÊNCIAS BIBLIOGRÁFICAS}

ALMEIDA, A. C. S.; SOUZA, J. L.; TEODORO, I.; BARBOSA, G. V. S.; MOURA FILHO, G.; FERREIRA JÚNIOR, R. A.. Desenvolvimento vegetativo e produção de variedades de cana-de-açúcar em relação a disponibilidade hídrica e unidades térmicas. Ciência e Agrotecnologia (UFLA), v. 32, p. 1441-1448, 2008.

BARBER, S. A.; CUSHMAN, J. H. Nitrogen up take model for agronomic crops. In: Modeling Waste Walter renovation - In: Land Treatment. 1. ed. New York: K. Iskardr. Wiley - interscience, p. 382-409. 1981.

BARBIERI, V.; SILVA, F. C. da; DIASAMBRONA, C. G. H. Modelagem de cana de açúcar para previsão de produtividade de canaviais no Brasil e na Austrália. CAI, v. 39, p. 745-762. 2010. 
BATISTA, E. L. D. S., ZOLNIER, S., RIBEIRO, A., LYRA, G. B., DA SILVA, T. G., \& BOEHRINGER, D. Modelagem do crescimento de cultivares de cana-de-açúcar no período de formação da cultura. Revista Brasileira de Engenharia Agrícola Ambiental, 17(10), 1080-1087, Campina Grande, 2013.

COSTA, L. G.; MARIN, F. R.; NASSIF, D. S. P.; PINTO, H. M. S.; LOPES-ASSAD, M. L. Simulação do efeito do manejo da palha e do nitrogênio na produtividade da cana-de-açúcar. Revista Brasileira de Engenharia Agrícola e Ambiental (Impresso), v. 18, p. 469-474, 2014.

GODOY, A. P. Modelagem de processos de acumulação de biomassa e de açúcar da cana-de-açúcar via sistemas nebulosos. 2007. Dissertação (Mestrado em Engenharia Elétrica) - faculdade de Engenharia Elétrica e Computação, Universidade Estadual de Campinas, Campinas, 2007.

INMAN-BAMBER, N. G. CANEGRO: its history, conceptual basis, present and future uses. In: Robertson, M. J. (Ed.) Research and modelling approaches to assess sugarcane production opportunities and constraints. University of Queensland, Brisbane, 1994. p. 31-34.

JONES, J. W.; MISHOE, W. K.; BOOTE, L. Introduction to simulation and modeling. In: The Seminar/Workshop on Computer Crop Simulation and Data Base management for Agrotecnology Transfer, coord. Food \& Fertilizer Thecnology Center, national Chung Hsing Universityand IBSNAT/USAID. Taaiwan, 21 p. (FFTC. Technical Bulletin, 100). 1987.

JUNGCLAUS, MARIN, F. R.; JONES, J. W.; ROYCE, F.; Suguitani, C.; Donzelli, J.L.; PALLONE FILHO, W. J.; Nassif, D. S. P. Parameterization and Evaluation of Predictions of DSSAT/CANEGRO for
Brazilian Sugarcane. Agronomy Journal (Print), v. 103, p. 297-303, 2011.

NASSIF, D. S. P.; MARIN, F. R.; REZENDE, R.; PELLEGRINO, G. Q. Parametrização e avaliação do modelo DSSAT/Canegro para variedades brasileiras de cana-de-açúcar. Pesquisa Agropecuária Brasileira, v. 47, p. 311-318, 2012.

OLIVEIRA, H. F.; MANTOVANI, E. C.; SEDIYAMA, G. C. Avaliação de modelos de estimativa de produtividade da cana-de-açúcar irrigada em Jaíba-MG. Revista Brasileira de Agricultura Irrigada, v. 7, p. 112-127, 2013.

OLIVEIRA, R. A.; SANTOS, R. S.; RIBEIRO, A.; ZOLNIER, S.; BARBOSA, M. H. P. Estimativa da produtividade da cana-deaçúcar para as principais regiões produtoras de Minas Gerais usando-se o método ZAE. Revista Brasileira de Engenharia Agrícola e Ambiental (Online), v. 16, p. 549 / 5-557, 2012.

ROSSETTI, L. A. Zoneamento agrícola em aplicações de crédito e securidade rural no Brasil: aspectos atuariais e de política agrícola. Revista Brasileira de Agrometeorologia, Passo Fundo, v. 9, n. 3, p. 386-399, dez. 2001. Número especial.

SANTOS, V. R. dos; FILHO, G. M.; ALBUQUERQUE, A. W. de; COSTA, J. P. V. da; SANTOS, C. G. dos; SANTOS, A. C. I. dos. Crescimento e produtividade agrícola de cana-de-acúcar em diferentes fontes de fósforo. Revista Brasileira de Engenharia Agrícola e Ambiental, v.13, p.389-396, 2009.

SCARPARI, M. S.; BEAUCLAIR, E. G. F. de. Sugarcane maturity estimation through edaphic-climatic parameters. 
Scientia Agricola. Piracicaba, v. 61, n. Agrícola). Universidade Federal de Viçosa, 5, p. 486-491, Sep./Oct. 2004. UFV.

SILVA, R. F. Calibração do modelo DSSAT/CANEGRO para a cana-deaçúcar e seu uso para a avaliação do impacto das mudanças climáticas. Viçosa, 2012. 56p. Dissertação (Mestrado em Meteorologia
SINGELS, A.; BEZIDENHOUT, C. N. A new method of simulating dry matter partioning in the DSSAT/CANEGRO sugarcane model. Field Crops Research, Amsterdam, v. 78, p. 151-164, 2002. 